\title{
Nonequilibrium properties of the one-dimensional hard-point gas system
}

\author{
Giulio Casati, ${ }^{1,2,3}$ Tomaž Prosen, ${ }^{4}$ Lei Wang, ${ }^{3}$ and Baowen $\mathrm{Li}^{3,5}$ \\ ${ }^{1}$ Center for Nonlinear and Complex Systems, Universita' degli studi dell'Insubria, Via Valleggio, 1122100 Como, Italy \\ and CNR-INFM \\ ${ }^{2}$ Istituto Nazionale di Fisica Nucleare, Sezione di Milano, Milano, Italy \\ ${ }^{3}$ Department of Physics and Center for Computational Science and Engineering, Faculty of Science, National University of Singapore, \\ Singapore 117542-46, Singapore \\ ${ }^{4}$ Physics Department, Faculty of Mathematics and Physics, University of Ljubljana, Ljubljana, Slovenia \\ ${ }^{5}$ Laboratory of Modern Acoustics and Institute of Acoustics, Nanjing University, 210093, China
}

(Received 19 April 2006; published 26 September 2006)

\begin{abstract}
We discuss the stability properties of a one-dimensional hard-point gas. We study the decay of the Loschmidt echo which describes the stability of the motion under system perturbations. We show a universal behavior in the echo decay which is intimately connected to the linear dynamical instability of the motion. In particular, in spite of such a weak instability, the asymptotic decay follows a simple exponential law.
\end{abstract}

DOI: 10.1103/PhysRevE.74.037201

PACS number(s): 05.45.Ac, 03.67.Lx, 05.45.Mt

The understanding of the statistical behavior of classical systems of finitely many particles from the underlying microscopic motion is one of the outstanding problems in statistical mechanics. The existing ergodic theory and the more recent achievements in nonlinear dynamical systems provide a sufficiently satisfactory frame for such understanding. In particular we have now a fairly good classification of different statistical properties: at the one end of the so-called ergodic hierarchy we have integrable systems - a prototype of which is the system of harmonic oscillators. At the opposite end we have the exponentially unstable systems like the gas of hard spheres with strong chaotic properties. However, in between these two extreme categories there is a rich variety of behavior which is the realm of most physical systems about which much less is known, our knowledge being mainly based on numerical experiments. In particular it would be highly interesting to have a physical model, in between the two above cases, for which clear statistical properties can be established so that it may serve as a firm ground for further investigations [1].

This is the purpose of the present paper and, to this end, the one-dimensional hard-point gas of alternating particles $m_{1}$ and $m_{2}$ appears to be a good candidate since it is known to exhibit linear instability and numerical evidence has been provided [2] for mixing behavior when the mass ratio $r$ $=m_{2} / m_{1}$ is an irrational number. The Hamiltonian of this system can be written as

$$
H=\sum_{j=1}^{N} \frac{p_{j}^{2}}{2 m_{j}}+V\left(q_{j+1}-q_{j}\right)
$$

where $q_{j}$ and $p_{j}$ are canonical coordinates and $V(q>0)=0$ and $V(q<0)=\infty$. In our computations we take $m_{2 j-1}=1$ and $m_{2 j}=r$ with $r=(\sqrt{5}+1) / 2$. Periodic boundary conditions are used; namely, we consider particles on a ring of circumference 1 , so that the mean spacing between each pair of particles is $a=\overline{q_{j+1}-q_{j}}=1 / N$. We fix the total (kinetic) energy $E=N$, and we measure time in terms of the integer number $n$ of all collisions up to a given instant.

Our interest is to answer some important questions such as how a small imperfection will influence the later fate of a system, how static errors or random fluctuations will change the motion and to what extent orbit instability can influence the decay rate of physical quantities. In this connection an important quantity is the sensitivity of the system's behavior to small perturbations in the Hamiltonian. It can be described by the so-called fidelity, or Loschmidt echo, which is defined as the overlap of two time evolving states (phase-space distributions) which, starting from the same initial condition, evolve under two slightly different Hamiltonians.

In the following, we formulate the problem in the language of many-particle Loschmidt echoes [3]; namely, we chose a specific (macroscopic) set $\mathcal{M}$ in a many-particle phase space. Then we prepare an ensemble of systems distributed uniformly in $\mathcal{M}$, propagated forward with unperturbed evolution, and propagated backward with the perturbed evolution for time $t$. The probability to return to the original set $\mathcal{M}$, or statistically, the relative fraction of the orbits arriving there, gives the fidelity $F(t)$. If $T_{n}(z)$ denotes the many-body map propagating the system for time $n$ starting from a phase space point $z$ and $\rho(z)$ denotes a characteristic function on the set $\mathcal{M}$, then the fidelity can be written as

$$
F_{\delta}(n)=\frac{1}{V} \int d^{2 N} z \rho\left[T^{(-n)}(z)\right] \rho\left[T_{\delta}^{(-n)}(z)\right],
$$

where $V$ is the phase-space volume of the set $\mathcal{M}$. For the perturbed evolution we take the same Hamiltonian (1) in which the mass of every other particle is slightly changedi.e., $m_{2 j-1}^{\prime}=1$ and $m_{2 j}^{\prime}=r+\delta$.

The behavior of classical fidelity has been recently discussed [3-8], largely motivated by previous results in the quantum domain [4,9-14] and by the possible use in quantifying stability of quantum computation [15]. In particular it has been shown [7] that for classically chaotic, exponentially unstable systems, the decay rate of fidelity is perturbation independent and, asymptotically, fidelity decays as correlation functions. However, very recently, a different universal behavior of classical Loschmidt echoes has been suggested for systems - the example being an abstract triangle map 


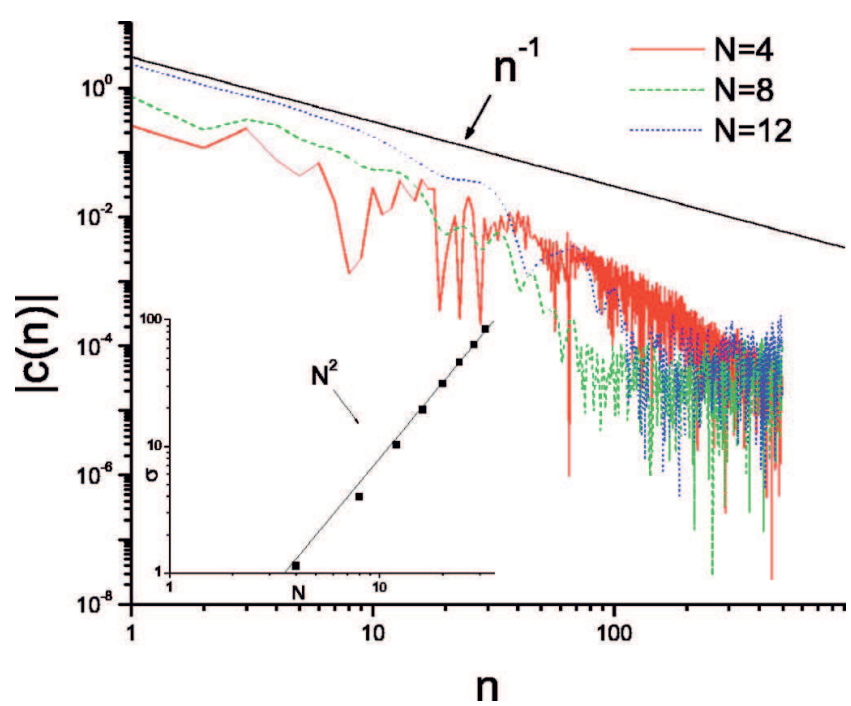

FIG. 1. (Color online) Correlation $c(n)$ versus time $n$. The solid line is $n^{-1}$. Inset: integrated correlation function $\sigma$ [Eq. (7)] versus particle number $N$. The data follow the $N^{2}$ law very well.

[16] — which lacks exponential instability but at the same time displays a mixing property [17]. Such dynamical behavior is sometimes called pseudochaos [1].

In this paper we present analytical and numerical evidence that the one-dimensional (1D) hard-point gas exhibits a universal scaling law for the fidelity decay characterized by a universal scaled time variable $|\delta|^{2 / 5} n$. This nicely confirms and generalizes to a many-body physical system, the results previously obtained for an abstract triangle map [16], and shows that the scaling behavior is a universal feature of the linear mixing property. Since our model is in fact a manybody system where the thermodynamic limit $N \rightarrow \infty$ can be studied, we also derive, using some mild statistical assumptions, the scaling of relevant physical quantities with the size $N$.

Let us first provide additional, convincing evidence for the mixing property of the 1D hard-point gas by demonstrating the decay of autocorrelation functions. To this end we plot in Fig. 1 the autocorrelation

$$
c\left(n^{\prime}\right)=\left\langle k(n) k\left(n+n^{\prime}\right)\right\rangle_{n}-\langle k(n)\rangle_{n}^{2},
$$

where

$$
k(n)=\sum_{j=1,3,5, \ldots} \frac{1}{2} m_{j} v_{j}^{2}(n)
$$

is the total kinetic energy $k(n)$ of all particles at $o d d$ positions at time $n$. It is seen that, for finite $N$, the asymptotic decay of $c(n)$ is at least as fast as $n^{-1}$. Note that this may be different from the hydrodynamic behavior of 1D hard-point gas in the thermodynamic limit $N \rightarrow \infty$ where a power law decay of correlations $t^{-\alpha}$, with $\alpha$ between 0.65 and 0.75 , has been typically observed $[18,19]$.

We turn now to the echo decay and discuss first orbit instability. We will make reference to the triangle map whenever needed. Indeed the triangle map contains the essential ingredients of the evolution of the hard-point gas, which may

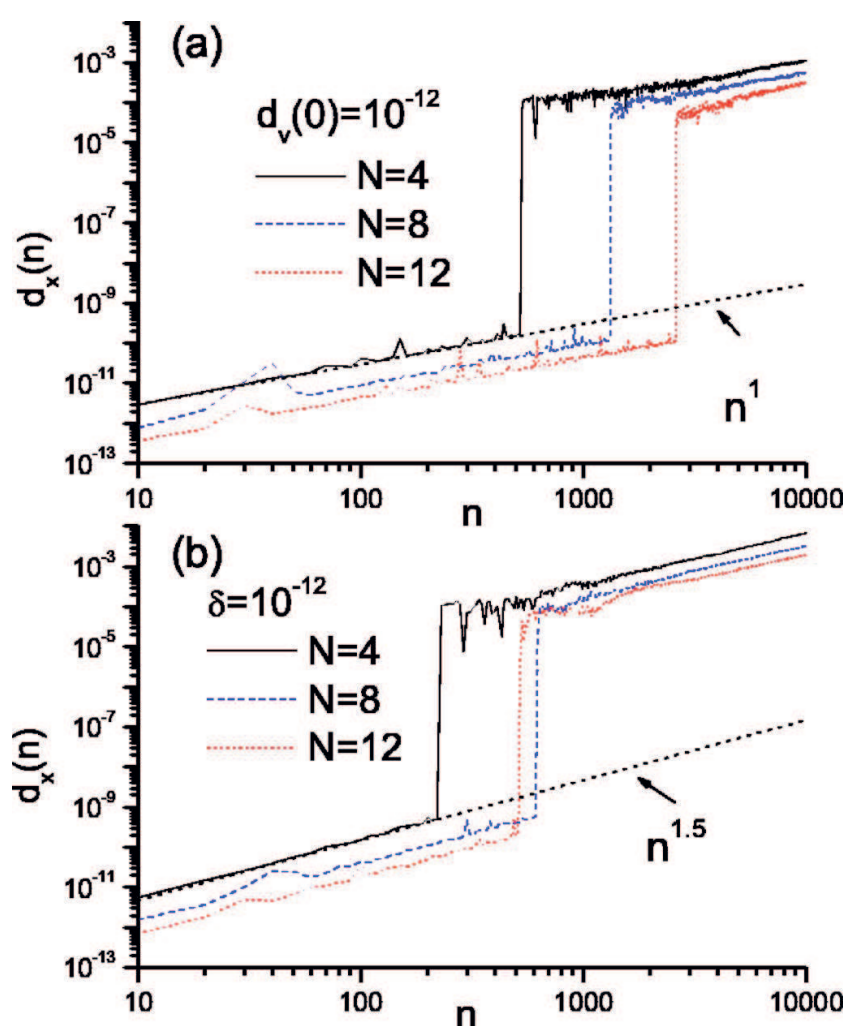

FIG. 2. (Color online) (a) Average distance in coordinate space $d_{x}(n)$ versus time $n$ for two nearby initial orbits of the unperturbed system. The orbits are initially separated in velocity space by the distance $d_{v}(0)=10^{-12}$, and the average is taken over $2 \times 10^{7}$ different initial conditions. The dotted line has slope 1. (b) Average distance versus time for two orbits starting from the same initial condition and evolving under the unperturbed and perturbed Hamiltonian respectively, with $\delta=10^{-12}$. The data are averaged over $2 \times 10^{7}$ different initial conditions. The dotted line has slope 1.5. Note that large vertical jumps in $d_{x}(n)$ observed in all data are due to occurnences of triple collisions for sufficiently long timesnamely, the fact that the two observed initially nearby orbits may experience a different sequence of collisions and hence become macroscopically different.

be considered as a high-dimensional billiard inside an $N$-dimensional polytope-namely, the free evolution and the discontinuous phase-space displacements which correspond to collisions of pairs of adjacent particles.

Let $x_{j}=q_{j+1}-q_{j} \geqslant 0$ denote the interparticle distance and let us consider the distance $\Delta x_{j}(n)=x_{j}^{\prime}(n)-x_{j}(n)$ of two orbits-say, $x_{j}(n)$ and $x_{j}^{\prime}(n)$-of two nearby Hamiltonians (separated by $\delta$ ). Assuming that collisions happen in a statistically uncorrelated and random way, we can write the diffusion law in momentum space as $\left[\Delta p_{j}(n)\right]^{2} \propto C \delta^{2}|n / N|$, with the constant $C$ independent of perturbation $\delta$, size $N$, and time $n$. The time is scaled by $N$ in order to account for the fact that a fraction of $1 / N$ collisions happen for the specified pair of particles $j$. Further, knowing that the linearized evolution is parabolic, we immediately have $\left[\Delta x_{j}(n)\right]^{2}$ $\propto C a^{2} \delta^{2}|n / N|^{3}$ (see Ref. [16] for a very similar argument). It follows that the coordinate space-Euclidean distancebetween the above pair of orbits scales as 


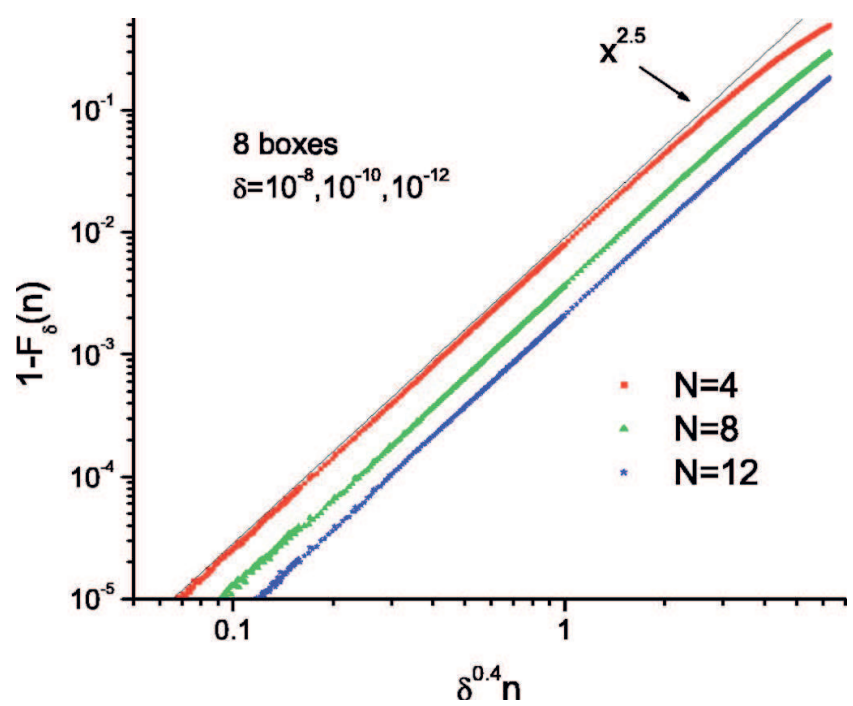

FIG. 3. (Color online) Short-time fidelity decay $1-F_{\delta}(n)$ versus $\delta^{2 / 5} n$. The solid line has slope 2.5 . In order to compute fidelity we divide the phase space into eight cells (boxes) as described in footnote 1 . We then take $2 \times 10^{7}$ initial points in the phase space and evolve these points with the perturbed Hamiltonian up to time $n$. Then we perform the reverse evolution with the unperturbed Hamiltonian for time $n$ and compute the fraction of points which fall again in the initial cell, after the total time $2 n$.

$$
d_{x}(n)=\sqrt{\sum_{j=1}^{N}\left[\Delta x_{j}(n)\right]^{2}} \approx \sqrt{N}\left|\Delta x_{j}(n)\right|=C|\delta| \frac{n^{3 / 2}}{N^{2}} .
$$

In Fig. 2 we present a numerical confirmation of this result together with the expected linear distance growth between two neighboring orbits of the same Hamiltonian.

Let us now derive the fidelity decay for short times and sufficiently small perturbation strength $\delta$. In this perturbative approximation, which is accurate as long as $1-F_{\delta}(n) \ll 1$, the fidelity can be written simply as the probability [16] that the orbits of the two evolutions (perturbed and unperturbed) follow the same history of collisions; i.e., they do not get separated by a triple-collision manifold. Thus to leading order in $\delta$ fidelity decays only due to a pair of collisions happening at different places (at the same time) for the two orbits. In other words, we write fidelity as one minus the total probability $P(n)$ that up to time $n$ at least one collision happens at a different place. We assume that due to the strong ergodic properties of the system [18], collisions happen pseudorandomly and uniformly. Then for a specific but arbitrary pair of particles—say, $j, j+1$-we have $P(n)=\sum_{n^{\prime}=0}^{n}\left|\Delta x_{j}\left(n^{\prime}\right)\right| / a$ and write the fidelity as

$$
F_{\delta}(n)=1-P(n)=1-\frac{3}{2} C|\delta| \frac{n^{5 / 2}}{N^{3 / 2}}
$$

The numerical results of Fig. 3 nicely confirm the scaling (6) and reproduce with high accuracy the theoretical time dependence.

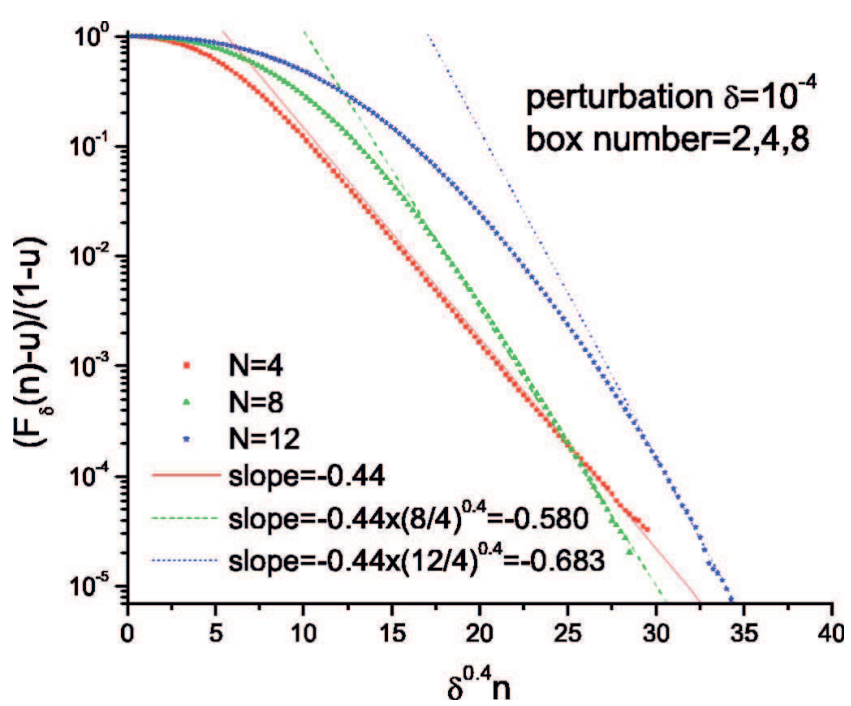

FIG. 4. (Color online) Long-time fidelity decay $\left[F_{\delta}(n)-u\right] /$ $(1-u)$ versus $\delta^{2 / 5} n$. Here $u \equiv V / V_{E}=1 / 2,1 / 4,1 / 8$ (for $N=4,8,12$, respectively) is the relative volume of the initial set $\mathcal{M}$ on the energy surface, giving the asymptotic value of fidelity. The straight lines give the expected asymptotic exponential decay with slopes which scale as $\propto N^{2 / 5}$. Here we take $4 \times 10^{9}$ intial conditions for $N=4$ and 8 . For $N=12$ we take $6 \times 10^{10}$ initial conditions in order to compute the fidelity for longer times. The scaling has been checked with different values of $\delta$.

For long times, we follow the general renormalization arguments of Ref. [16]. Namely, in the nonperturbative regime, fidelity should be a universal function of a single parameter $F_{\delta}(n)=\phi\left(|\delta|^{2 / 5} \sigma^{1 / 5} n\right)$. Here $\sigma$ is the integrated correlation function of the perturbation of the Hamiltonian

$$
\sigma=\frac{1}{2} \sum_{n=-\infty}^{\infty} c(n)
$$

The numerical reults in Fig. 1 show that $\sigma$ is indeed finite and grows with $N$ as $\sigma \propto N^{2}$. This is a simple consequence of the fact that the kinetic energy is an extensive quantity $k(n) \propto N$ implying $c(0) \propto N$. If in addition we assume that $c(n)$ decays faster than $1 / n$, within a time scale of $N$ collisions (or a collision per particle), then the quantity $\sigma$ appearing in the scaling parameter is $\sigma=D N^{2}$, where $D$ is some constant independent of $\delta, n$, and $N$.

The numerical results in Fig. 4 show a very good agreement with the theoretically predicted scaling (on $n, N$, and $\delta$ ) and give the long-time asymptotic $\phi(n)=\exp (-n)$. Hence we have a prediction for the scaling of long-time fidelity decay of the hard-point gas:

$$
F_{\delta}(n)=\exp \left(-C^{\prime}|\delta|^{2 / 5} N^{2 / 5} n\right)
$$

where $C^{\prime}$ is a numerical constant independent of $\delta, n$, and $N$.

In order to numerically compute the Loschmidt echo, the energy surface, or the momentum space, is divided into 
either two, four, or eight cells of equal size, ${ }^{1}$ and one of them is chosen as our set $\mathcal{M}$ with known volume $V$. Initially, $N$ particles (we take $N=4,8$, and 12) are randomly placed on a ring of unit length. The velocities of the particles are also randomly chosen while the total kinetic energy is fixed to unity. Then the particles move freely unless elastic collisions happen. We evolve each initial state up to $n$ collisions, then reverse all the velocities and evolve the system again but with the perturbed mass distribution: $m_{2 j-1}=1$ and $m_{2 j}$ $=r+\delta$, again up to $n$ collisions. Then we compute fidelity as

\footnotetext{
${ }^{1}$ In order to keep numerical experiments as simple as possible, we divide the energy surface, or momentum space, into $n$ cells of equal volume $u=V / V_{E}=1 / n$. The case $n=2$ is trivial: the two cells are defined by $v_{i}>0$ and $v_{i}<0$, for any particle $i$ where $v_{i}=p_{i} / m_{i}$. However, since the total momentum and the total kinetic energy are conserved and because we measure time as the total number of collisions, it is not trivial to uniformly divide into a larger number of cells. There is, however, a simple solution for the division into $n=2,4,8$ cells provided the number of particles is a multiple of 4 . Indeed let us take four vectors

$$
\left(\begin{array}{l}
\vec{e}_{1} \\
\vec{e}_{2} \\
\vec{e}_{3} \\
\vec{e}_{4}
\end{array}\right)=\left(\begin{array}{ccccc}
1 & \sqrt{r} & 1 & \sqrt{r} & \ldots \\
1 & 0 & -1 & 0 & \ldots \\
0 & 1 & 0 & -1 & \ldots \\
\sqrt{r} & -1 & \sqrt{r} & -1 & \ldots
\end{array}\right)
$$

and the additional vector

$$
\vec{s}=\left(\begin{array}{lllll}
v_{1} & \sqrt{r} v_{2} & v_{3} & \sqrt{r} v_{4} & \cdots
\end{array}\right)^{\dagger} .
$$

Notice that $\vec{e}_{1} \cdot \vec{s}$ is the total momentum which is always conserved and may be trivially set to zero. Now take $\lambda_{i}=\vec{e}_{i} \cdot \vec{s}, i=2,3,4$. Then the conditions $\lambda_{i}>0, \lambda_{i}<0, i=2,3,4$, and their combinations divide the momentum space to 2,4 , and 8 cells equally.
}

the fraction of states which fall again in the initial cell $\mathcal{M}$.

We would like to stress that the results presented in this paper remain qualitatively the same for random (noisy) perturbation of masses (after each collision we choose a different uncorrelated random value of $\delta$ ). This is consistent with our analytical arguments which only require decay of temporal correlations and thus work even better in the case of noisy perturbation.

In conclusion, we have discussed the stability properties of an important example of classical many-body systems with intermediate, neither integrable nor chaotic dynamicsnamely, the 1D hard-point gas. By means of analytic calculations and numerical simulations we have derived two universal regimes of fidelity decay, for short and long times, both being characterized by a universal scaled time variable $|\delta|^{2 / 5} n$.

In particular our results provide clear empirical evidence that in the presence of small imperfections, the accuracy of observable quantities can decrease exponentially with time even though errors of trajectories propagate in time only linearly or according to a power law. In our opinion this is a remarkable property which calls for the attention of the mathematical community. In some sense this behavior resembles the situation in quantum mechanics, where, apart from the Ehrenfest time, logarithmically short in $\hbar$, there is no exponential instability [20].

We acknowledge financial support by the PRIN 2006 "Effects of decoherence and imperfections on quantum computation and quantum simulation with trapped particles" (GC), by Grants Nos. P1-0044 and J1-7347 of the Slovenian Research Agency (TP), by a Faculty Research Grant of NUS, and by DSTA, Singapore, under Project Agreement No. POD0410553 (BL).
[1] O. Lyubomudrov, M. Edelman, and G. M. Zaslavsky, Int. J. Mod. Phys. B 17, 4149 (2003).

[2] G. Casati and J. Ford, J. Comput. Phys. 20, 97 (1976)

[3] G. Veble and T. Prosen, Phys. Rev. E 72, 025202(R) (2005).

[4] T. Prosen and M. Žnidarič, J. Phys. A 35, 1455 (2002).

[5] G. Benenti and G. Casati, Phys. Rev. E 65, 066205 (2002).

[6] B. Eckhardt, J. Phys. A 36, 371 (2003).

[7] G. Benenti, G. Casati, and G. Veble, Phys. Rev. E 67, 055202(R) (2003).

[8] G. Veble and T. Prosen, Phys. Rev. Lett. 92, 034101 (2004).

[9] A. Peres, Phys. Rev. A 30, 1610 (1984).

[10] H. M. Pastawski et al., Physica A 283, 166 (2000).

[11] R. A. Jalabert and H. M. Pastawski, Phys. Rev. Lett. 86, 2490 (2001).

[12] T. Prosen, Phys. Rev. E 65, 036208 (2002).

[13] Ph. Jacquod et al., Phys. Rev. E 64, 055203(R) (2001).
[14] N. R. Cerruti and S. Tomsovic, Phys. Rev. Lett. 88, 054103 (2002).

[15] G. Benenti, G. Casati, and G. Strini, Basic Concepts Principles of Quantum Computation and Information, Vol. I (World Scientific, Singapore, 2004); M. A. Nielsen and I. L. Chuang, Quantum Computation and Quantum Information (Cambridge University Press, Cambridge, England, 2001).

[16] G. Casati, T. Prosen, J. Lan, and B. Li, Phys. Rev. Lett. 94, 114101 (2005).

[17] G. Casati and T. Prosen, Phys. Rev. Lett. 83, 4729 (1999); 85, 4261 (2000).

[18] G. Casati and T. Prosen, Phys. Rev. E 67, 015203(R) (2003).

[19] P. Grassberger, W. Nadler, and L. Yang, Phys. Rev. Lett. 89, 180601 (2002).

[20] V. Latora and M. Baranger, Phys. Rev. Lett. 82, 520 (1999). 